\title{
Differential heart-rate conditioning in the rabbit: Failure to find a specific pre-US response $^{1}$
}

RUSSELL A. LOCKHART and DAVID C. STEINBRECHER, University of California, Santa Barbara, Calif. 93106

Differential heart-rate conditioning was studied in rabbit Ss under three conditions of delay (.5, 5, and $10 \mathrm{sec})$. Significant differential conditioning $/ C R=$ deceleration) occurred in the .5-sec condition, to a greater degree in the 5-sec condition, and failed to develop in the 10 -sec condition. Of primary interest was the failure to observe the development of a pre-US response separate from the deceleration triggered by the $C S$, a result required by the assumption of pre-US responding mediated by verbal mechanisms.

In studies of human classical conditioning, it is not uncommon to observe what is described as the multiple response phenomenon (Lockhart, 1966a). This is principally true in situations where a relatively long CS-US interval is employed. The phenomenon consists (in a variety of autonomic response systems, but perhaps most clearly in the GSR) of a response to CS onset (the CS-R), a subsequent response(s) prior to the onset of the US (the pre-US-R), and yet further responding during the normal UR interval when the US is withheld. Early interpretation of these distinct responses (Stern, Stuart, \& Winokur, 1960; Stewart, Stern, Winokur, \& Fredman, 1961) described the initial response as an orienting response, the response(s) antedating US onset as true conditioned anticipatory responses, and responses occurring in the absence of US as disparity responses (i.e., responding to the nonconfirmation of an anticipated stimulus).

More recently, the phenomenon of multiple responding appears to offer a potential methodology for the study of the role of cognitive processes as they operate in human classical conditioning. In particular, it has been suggested (Lockhart, $1966 \mathrm{~b}, 1970)$ that the pre-US response may be the most sensitive indicator of "awareness" of the contingent relations between CS and US, and this notion has received support in recent studies of the relation between cognitive processes and multiple responses (e.g., Fuhrer \& Baer, 1969). If the genesis of pre-US responding (as distinct from CS responding and/or the inhibition of delay of CS-Rs) rests critically in verbal-perceptual mechanisms of the type reflected in techniques of assessing verbal awareness, it may be predicted that pre-US responding would be affected by damage to verbal-perceptual mechanisms and perhaps be absent in nonhuman organisms lacking verbal mechanisms entirely. It has been shown that pre-US responding is absent in severely retarded Ss, occurs with greater frequency in less severely retarded Ss, and is typical of the college student population (Grings, Lockhart, \& Dameron, 1962; Lockhart \& Grings, 1964; Lockhart, 1966b).

The purpose of the present experiment was to search specifically for the occurrence of a distinct pre-US response in an animal S. Rabbits were exposed to a differential conditioning paradigm under different interstimulus intervals, with heart rate as the observed response system. METHOD AND PROCEDURE

Twelve New Zealand albino rabbits were divided randomly into three groups of four Ss and subsequently conditioned with an interstimulus interval (ISI) of $.5,5$, or $10 \mathrm{sec}$ in a differential conditioning paradigm, with US delivered simultaneously with CS offset. Tones of 200 and $3,000 \mathrm{~Hz}, 20 \mathrm{~dB}$ above sound level, were employed as CSt and CS(alternated across animals). US was a .5-sec dc shock delivered to the animal's left ear. Two conditioning sessions were employed separated by $24 \mathrm{~h}$. During the first session, each animal received $10 \mathrm{CS}+$ and $10 \mathrm{CS}-$ adaptation trials intermixed with two unpaired US trials. Adaptation was followed by an acquisition phase consisting of $40 \mathrm{CS}+$ and $40 \mathrm{CS}-$ randomly mixed trials. Of the $40 \mathrm{CS}+$ trials, 30 were paired with US at the appropriate ISI and 10 served as acquisition test trials. Adjacent CS- trials served as comparison stimuli. During the second session, each animal received an acquisition series identical to the first series. Intertrial intervals averaged $2 \mathrm{~min}$ and varied between 1 and $3 \mathrm{~min}$.

Heart rate was recorded from three stainless steel electrodes subcutaneously implanted (two thorasic, one back). The EKG signal was filtered, amplified, and fed to a cardiotachometer with a range of 60 to $900 \mathrm{bpm}$. Both EKG and cardiotachometer output were recorded on a Beckman Type $\mathbf{R}$ dynograph.
Analysis of heart rate occurred in the following steps: (1) Each beat represented in the cardiotachometer tracing was measured and translated into bpm; (2) the resulting bpm values for each beat were then submitted to computer analysis that transformed these values into $\mathrm{bpm} / \mathrm{sec}$. Essentially, this represents the averaged rate for each second, and the data were printed out in this form. This procedure was accomplished for pre-CS, CS, and post-CS intervals in each group on all trials for each animal.

RESULTS AND DISCUSSION

In virtually every case, the heart-rate response took the form of a rapid and generally sustained deceleration on acquisition test trials. The UR was characterized by an initial deceleration followed by a sizable acceleration. Across
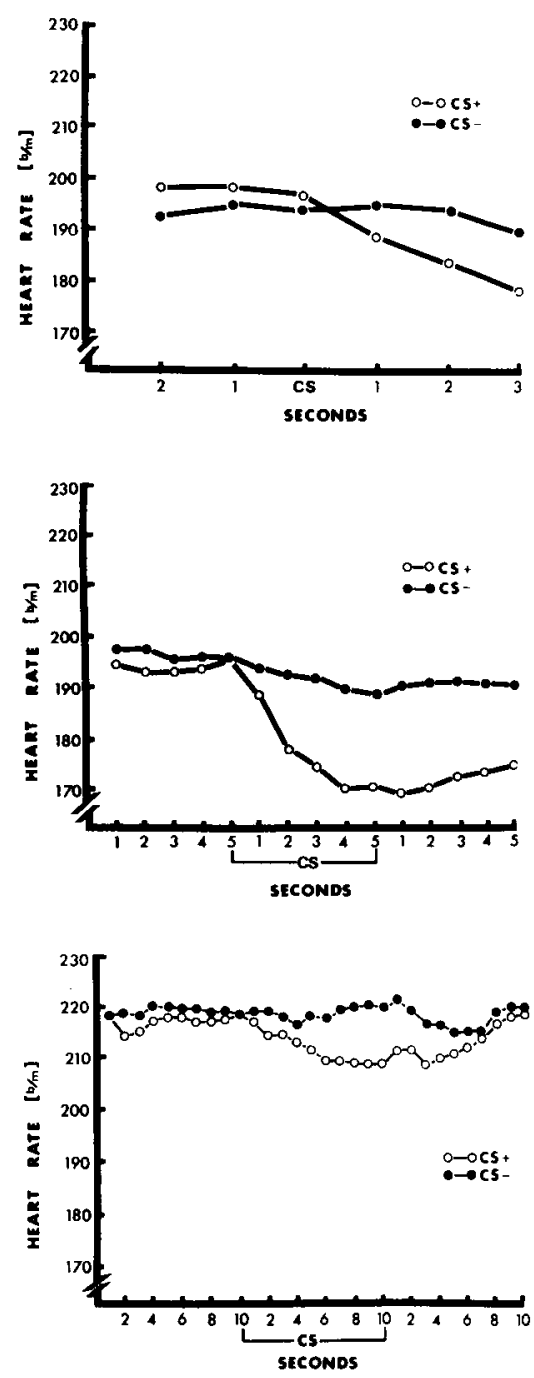

Fig. 1. Mean heart rate (for each second) in bpm for CS+ and CS - stimuli in three ISI conditions $($ top $=.5 \mathrm{sec} ;$ middle $=5 \mathrm{sec}$; bottom $=10 \mathrm{sec}$ ). Day 2 data only. 
days, the difference between $\mathrm{CS}+$ and $\mathrm{CS}-$ responses increased considerably. Figure 1 illustrates second-by-second rates of responding averaged over $10 \mathrm{CS}+$ and 10 CS - trials during the second session. Data for the first session are of the same form but with smaller differences by approximately one-half in each group.

In each case, the form of the response to CSt is deceleration. These curves are strikingly representative of individual curves, with little or no distortion in form resulting from such grouping. In each group, the deceleratory response begins within $1 \mathrm{sec}$ of CS onset. The deceleration increases to the 3 rd second in the $.5-\mathrm{sec}$ group, to the 5 th or 6 th second in the 5 -sec group, and to the 7th second in the 10-sec group. Following these points of maximum deceleration, there is a period of rate maintenance followed by an acceleratory phase tending toward pre-CS levels.

In order to assess the significance of these changes and particularly the differential response between CS+ and CS-, a value was computed for each test, and control comparison based on the change from the last pre-CS second to the point of maximum inflection during the period measured. These values for CSt and CS- are given in Table 1 for each animal by group. The difference between CS+ and CS- values are also presented and accompanied by individual $t$-test values and associated probability values.

This analysis indicates that differential conditioning failed to occur in any 10-sec animal, occurred in all 5 - sec animals, and three of four .5-sec animals. Further statistical analysis indicated that the overall degree of differential conditioning was significantly greater in the 5 -sec group than

Table 1

Mean Difference Between $\mathrm{CS}+$ and $\mathrm{CS}-$ in Terms of Maximum Deceleratory Response. Days 1 and 2 Combined.

\begin{tabular}{|c|c|c|c|c|c|}
\hline ISI & Animal & $\mathrm{CS}+$ & $\mathrm{CS}-$ & $\Delta$ & $\mathrm{t}$ \\
\hline \multirow[t]{4}{*}{.5} & 106 & 12.38 & 6.97 & 5.41 & $2.67^{*}$ \\
\hline & 108 & 23.15 & 7.23 & 15.92 & $2.42 *$ \\
\hline & 110 & 19.85 & 9.05 & 10.80 & $3.50 * *$ \\
\hline & 112 & 13.10 & 8.01 & 5.09 & 1.46 \\
\hline \multirow[t]{4}{*}{5} & 100 & 22.24 & 9.85 & 12.39 & $5.06 * *$ \\
\hline & 102 & 22.33 & 11.03 & 11.30 & $3.34 * *$ \\
\hline & 104 & 20.54 & 9.96 & 10.60 & $2.88 * *$ \\
\hline & 105 & 25.13 & 7.66 & 17.46 & $5.15^{* *}$ \\
\hline \multirow[t]{4}{*}{10} & 107 & 19.48 & 18.77 & .71 & .12 \\
\hline & 109 & 24.76 & 23.41 & 1.69 & .29 \\
\hline & 111 & 16.91 & 11.84 & 5.07 & 1.40 \\
\hline & 113 & 12.03 & 13.47 & -1.44 & .36 \\
\hline
\end{tabular}

Fig. 2. Heart rate (in bpm) by second for CS+ and CS- stimuli during four ordinally consecutive test (and control) trials in Animal 105 (5-sec ISI). Day 2 data only. in either the $.5-\mathrm{sec}$ or $10-\mathrm{sec}$ group, and significantly larger in the .5 -sec group than in the $10-\mathrm{sec}$ group. It is important to note that the failure of differential responding in the 10-sec group was not the result of failure of deceleration during CS+ (which approximated the degree of deceleration in the other two ISI conditions). Rather, a deceleration of approximately equal extent occurred during CS-. This is interpretable as a failure of inhibition during $\mathrm{CS}-$ with the suggestion that as ISI passes some optimal point it is the inhibitory mechanisms that are disrupted by temporal delay. ${ }^{2}$

On a group basis (as evident in Fig. 1), there exists no evidence for "multiple" responses. The form of the response was consistently an immediate deceleration increasing to or near the point of normal US occurrence (in the $5-\mathrm{sec}$ and $10-\mathrm{sec}$ groups) followed by a gradual increase toward the pre-CS rate level.

Is there evidence for multiple responding on a trial-by-trial (as opposed to averages across trials) basis? Figure 2 illustrates CS+ and $\mathrm{CS}-$ responding for Animal 105 during the last quarter of the second acquisition session. This animal was selected because he demonstrated the highest level of overall differential responding (on Day 1 as well). Determination of the existence of multiple responding is difficult because of the lack of criteria defining a heart-rate response. Quite obviously, the overall deceleration to
CS+ is a marked response. The problem lies in determination of the status of such complex responses as changes in rate of deceleration (or acceleration), or in the rate of return to a prestimulus base level. If the heart rate is considered a system sensitive to both central and peripheral factors, some of . which are reflexive responses to changes induced by experimental variables, then these complex aspects of the heart rate must be considered relevant to the question of multiple responding. For example, it would be desirable to distinguish a reflexive compensatory acceleration from an acceleration induced by a central process of perceived imminence of a noxious event. The data in Fig. 2 indicate only minor and subtle changes in rate and certainly are paled by comparison with the dramatic multiple changes in heart rate as observed in human Ss (e.g., Headrick \& Graham, 1968). There is, therefore, little evidence in these data for the existence of a multiple response pattern and particularly for the existence of a pre-US response separate from the large initial deceleration triggered by stimulus onset.

\section{REFERENCES}

FUHRER, M. J., \& BAER, P. E. Cognitive processes in differential GSR conditioning: Effects of a masking task. American Joumal of Psychology, 1969, 82, 168-180.

GRINGS, W. W., LOCKHART, R. E., \& DAMERON, L. E. Conditioning autonomic responses of mentally abnormal individuals.

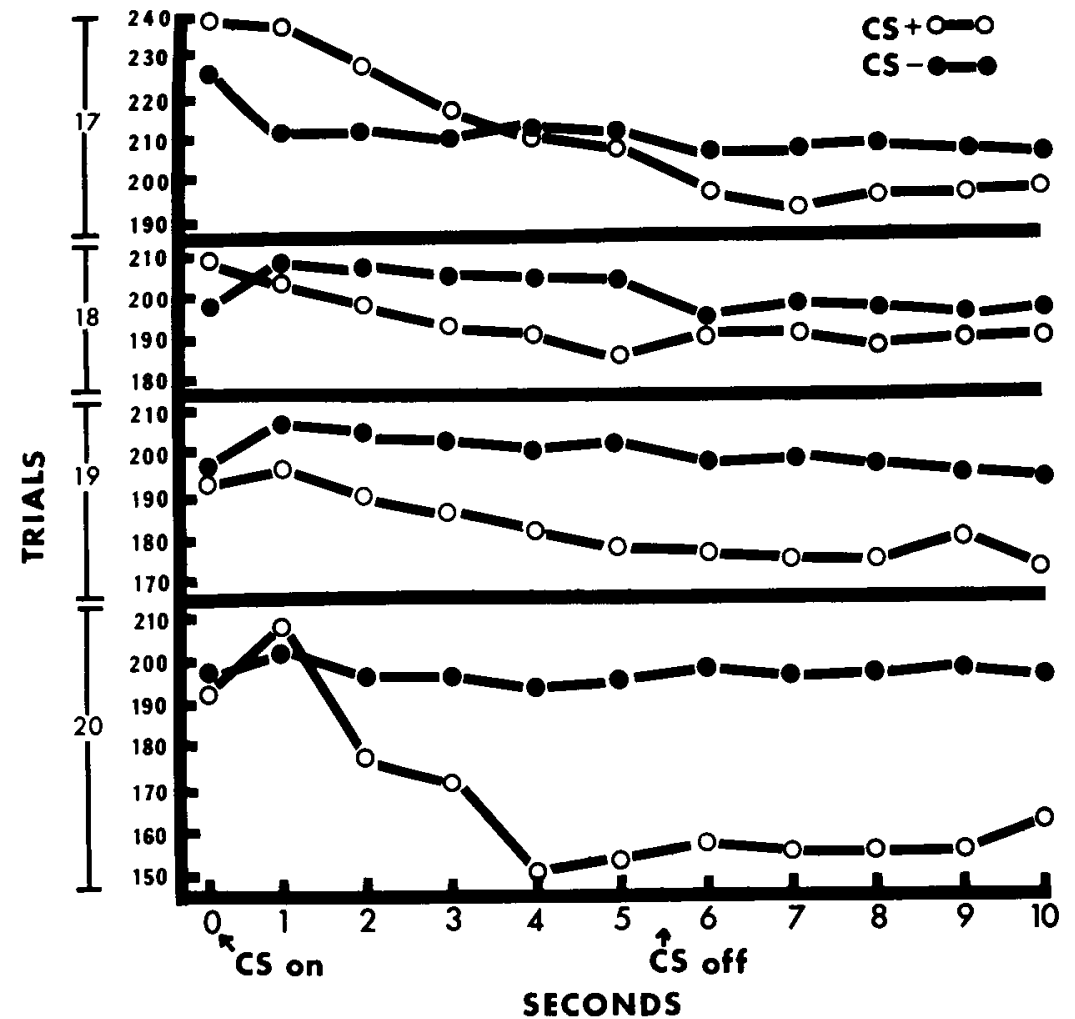


Psychological Monographs, 1962, 76(39), Whole No. 558.

HEADRICK, M. W., \& GRAHAM, F. K. Multiple component heart rate responses conditioned under paced respiration. Journal of Experimental Psychology, 1969, 79, 486-494.

LOCKHART, R. A. Comments regarding multiple response phenomena in long interstimulus interval conditioning. Psychophysiology, 1966a, 3, 108-114.

LOCKHART, R. A. Recent developments in the classical conditioning of autonomic responses. Symposium on Higher Nervous Activity, Fourth World Congress of Psychiatry, Madrid, Spain, 1966b.

LOCKHART, R. A. Distinguishing component processes reflected in autonomic behavior of human subjects during classical conditioning. International Journal of Psychobiology, 1970, in press.

LOCKHART, R. A., \& GRINGS, W. W. Interstimulus interval effects on GSR discrimination conditioning. Journal of Experimental Psychology, 1964, 67, 209-214. STERN, J. A., STEWART, M. A., \& WINOKUR, G. An investigation of some relationships between various measures of galvanic skin response. Joumal of Psychosomatic Research, 1961, 5, 215-223.

STEWART, M. A., STERN, J. A., WINOKUR, G., \& FREDMAN, S. An analysis of GSR conditioning. Psychological Review, 1961, 68, 60-67.

\section{NOTES}

1. This investigation was supported by a grant from USPHS (MH 12879-02). We wish to thank Larry Walrath, Mary Kidd, and Martha Cummings for their helpful assistance.

2. This point should not be overly stressed since differential responding in the 10-sec condition approached significance during Day 2 (see Fig. 1). During Day 2 (particularly in the late trials), responding to $\mathrm{CS}-$ changed from deceleration to a slight acceleration.

\section{Agonistic behavior in mice: Strain differences as a function of test illumination ${ }^{1}$}

\section{T. W. KLEIN, J. HOWARD, and J. C. DeFRIES, University of Colorado, Boulder, Colo. 80302}

In each of two experiments, C57BL (pigmented) mice were paired with members of the $B A L B / c$ (albino) strain under either high or low illumination and their agonistic behovior observed. In both experiments, under high illumination, C57BL Ss won approximately $90 \%$ of the bouts that resulted in submission. In contrast, under low illumination, C57BL mice won about $40 \%$ of the bouts, resulting in highly significant interactions between strain and level of test illumination. These results may explain the apparent inconsistency in the literature concerning the agonistic behavior of these two inbred strains.

McClearn (1967) has recently discussed the utility of inbred strain comparisons in behavioral research: For each of several strains, independent investigations have provided a profile for a variety of behaviors that are stable over time and space. These strain profiles are cumulative and permit characterization of members of a given strain for a number of traits. However, differential responses of different genotypes to changes in environmental conditions have also been reported. For example, differential effects of test illumination on the open-field behavior of albino and pigmented mice are well documented (McClearn, 1960; DeFries,
Hegmann, \& Weir, 1966; McReynolds, Weir, \& DeFries, 1967). When apparent inconsistencies in strain profiles are found, the possibility of a genotype-environment interaction should be considered.

Inbred strains have been widely used in studies of aggressive behavior in mice (Lagerspetz, 1964). Characteristically, one male from each of two inbred strains is placed in a neutral cage and agonistic behavior is observed. Using this procedure, Ginsburg \& Allee (1942) studied the effects of conditioning on social dominance in inbred strains of mice. In their initial experiments, they found that members of the C57BL strain were more aggressive than those of the $\mathrm{C} 3 \mathrm{H}$ strain, both of which were more aggressive than the $\mathrm{C}$ albino strain (BALB/c). In these experiments, a relatively high level of illumination was employed (a 60-W lamp placed $19 \mathrm{in}$. above the floor of the test cage). In a recent review of social behavior in the laboratory mouse, Wimer \& Fuller (1966) indicate that several researchers have subsequently used $B A L B / c$ mice as relatively nonaggressive Ss.

In contrast to the findings of Ginsburg \& Allee (1942), Scott (1942) has described the C57BL/10 strain as being "pacifist." Similarly, Tellegen, Horn, \& Legrand (1969) reported that C57BL/6J mice rarely fight vigorously when attacked; thus, they were suitable "standard victims" in an experiment to test the reinforcing properties of opportunity for aggression by BALB/cJ mice. Casual observation of Ss in experiments concerning social dominance in our laboratory suggest that $\mathrm{BALB} / \mathrm{c}$ (albino) mice are more aggressive than C57BL (pigmented) mice under relatively low levels of illumination. The primary objective of the present study was to test the hypothesis that level of test illumination will differentially influence the agonistic behavior of $B A L B / c$ and C57BL mice, which may account for the apparent inconsistency in the literature regarding the social dominance of these two inbred strains.

\section{METHOD}

In an initial experiment, six males from each of two inbred strains (BALB/cIbg and C57BL/lbg) were tested under each of two levels of illumination. The high-illumination condition was similar to that employed by Ginsburg \& Allee (a $60-\mathrm{W}$ incandescent bulb suspended 19 in. above the floor of the test cage), whereas low illumination was provided by a dim red light. Ss in this experiment were not experimentally naive; however, mice were individually housed in separate cages for at least 3 weeks prior to the beginning of the experiment. Each S (approximately 100 days of age) participated in one bout on each of six successive evenings. A bout consisted of placing two male mice in a stainless steel cage $(12 \times 8 \times 4$ in. $)$ separated by a partition for a period of $5 \mathrm{~min}$. The partition was then raised and the Ss were observed for $20 \mathrm{~min}$ through a clear Plexiglas cover. Bouts were terminated prior to $20 \mathrm{~min}$ if the fight was severe enough to produce bleeding. Within illumination condition, each BALB mouse was successively paired with every C57BL mouse, resulting in a total of 36 bouts for each condition.

In a subsequent experiment, several alterations in procedure were adopted: Experimentally naive $S s$ (individually housed for at least 75 days) were administered fighting experience in an attempt to increase the frequency of aggressive behavior. Each $\mathrm{S}$ (ranging in age from 99 to 125 days) was randomly paired for 20 bouts over a 4 -week period prior to final testing. In this experiment, the high-illumination condition was the same as that described above. However, low illumination was provided by raising the $60-\mathrm{W}$ bulb to approximately $8 \mathrm{ft}$ above the floor of the test cage. This resulted in levels of illumination of about 45- and 3-fc incident light for the high and low conditions, respectively. Training experience and final testing were conducted under the same levels of illumination.

The classical submissive posture (rearing back on hind legs with one foreleg drawn close to the body and the other stiffly 